\title{
Decision Analysis of Dynamic Spectrum Access Rules
}

\author{
GlobeCom
}

\author{
Juan D. Deaton \\ Christian Wernz \\ Luiz A. DaSilva
}

December 2011

The INL is a

U.S. Department of Energy

National Laboratory

operated by

Battelle Energy Alliance

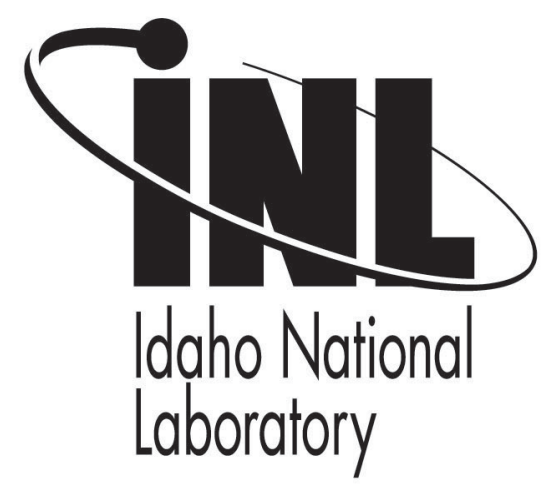

This is a preprint of a paper intended for publication in a journal or proceedings. Since changes may be made before publication, this preprint should not be cited or reproduced without permission of the author. This document was prepared as an account of work sponsored by an agency of the United States Government. Neither the United States Government nor any agency thereof, or any of their employees, makes any warranty, expressed or implied, or assumes any legal liability or responsibility for any third party's use, or the results of such use, of any information, apparatus, product or process disclosed in this report, or represents that its use by such third party would not infringe privately owned rights. The views expressed in this paper are not necessarily those of the United States Government or the sponsoring agency. 


\section{Decision Analysis of Dynamic Spectrum Access Rules}

\author{
*N\&HS Directorate \\ Idaho National Lab \\ Idaho Falls, Idaho USA
}

\author{
Juan D. Deaton*†, Christian Wernz ${ }^{\S}$, Luiz A. DaSilva ${ }^{\ddagger \dagger}$ \\ $\dagger$ Bradley Dept. of Electrical and Computer Engineering \\ $\S$ Grado Dept. of Industrial and Systems Engineering \\ Virginia Tech \\ Blacksburg, Virginia USA
}

\author{
$\ddagger$ CTVR \\ Trinity College \\ Dublin, Ireland
}

\begin{abstract}
A current trend in spectrum regulation is to incorporate spectrum sharing through the design of spectrum access rules that support Dynamic Spectrum Access (DSA). This paper develops a decision-theoretic framework for regulators to assess the impacts of different spectrum access rules on both primary and secondary operators. We analyze access rules based on sensing and exclusion areas, which in practice can be enforced through geolocation databases. Our results show that receiver-only sensing provides insufficient protection for primary and co-existing secondary users and overall low social welfare. On the other hand, using combining sensing information of the transmitter and receiver of a communication link provides dramatic increases in system performance. The performance of using these link end points is relatively close to that of using many cooperative sensing nodes associated to the same access point and large link exclusion areas. These results are useful to regulators and network developers in understanding in developing rules for future DSA regulation.
\end{abstract}

\section{INTRODUCTION}

Recent trends in regulatory paradigms have been shifting from the traditional command and control model of spectrum management to one of shared use through Dynamic Spectrum Access (DSA) [1-3]. Both the Federal Communications Commission (FCC) and Ofcom have released initial regulation for unlicensed used of TV white space devices, and the FCC, in a recent Notice of Inquiry (NOI), is requesting information on the viability of DSA techniques [3]. In this effort, the FCC seeks to understand how spectrum access rules based on spectrum sensing and geolocation databases can be useful in providing secondary operators with opportunistic access while protecting primary users from harmful interference.

Our paper applies decision theory to analyze the impact of different shared use spectrum access rules. This decision

This work is supported by the Idaho National Laboratory (INL) through the Laboratory Directed Research \& Development (LDRD) Program under DOE Idaho Operations Office Contract DE-AC07-05ID14517. The United States Government retains and the publisher, by accepting the article for publication, acknowledges that the United States Government retains a nonexclusive, paidup, irrevocable, worldwide license to publish or reproduce the published form of this manuscript, or allow others to do so, for United States Government purposes. The views and conclusions contained in this document are those of the authors and should not be interpreted as representing the official policies, either expressed or implied, of the Department of Energy or the U.S. Government. This material is also based upon works supported by the Science Foundation Ireland under Grant No. 10/IN.1/I3007. analysis provides regulators with a methodology to evaluate spectrum access rules based on the resulting utility to the various constituencies that are vying for spectrum. Our first contribution in this study is a multi-attribute utility model for evaluating spectrum access rules. This multi-attribute utility model is based on the fundamental objectives of primary and secondary operators deploying DSA. Through the Analytical Hierarchy Process (AHP) [4], we weight the relative importance between objectives, using input from experts in network performance. In our second contribution, we propose and evaluate nine spectrum access rules based on spectrum sensing and geolocation databases. Here, we simulate a scenario in which a primary operator shares spectrum with a secondary operator. The results of the simulation are then evaluated through our multi-attribute utility model and we calculate the utility of each rule.

Our paper is organized as follows. Section II defines the anatomy of a spectrum access rule $\mathcal{R}$. Section III describes the decision analytic framework. In this section, we propose an objective hierarchy, develop the multi-attribute utility model, and identify nine spectrum access rules that we set out to evaluate in the ensuing analysis. Section IV presents our system model, simulation scenario, and the specific implementation of our utility model. In Section V, we present the resulting utilities from our simulation using the multi-attribute utility model. We conclude this paper, in Section VI, with the summary of our results and a discussion future work.

\section{Spectrum Access Rules}

Recent regulations in [1] and [2] for DSA describe the set of underlying behaviors (sub-rules) that DSA radios use to opportunistically access primary spectrum. These regulations can be categorized into three sub-rules: exclusion $(\mathcal{E})$, channel assignment $(\mathcal{A})$, and power and transmission control $(\mathcal{P})$. Thus, when a DSA radio accesses the spectral medium it uses the spectrum access rule defined by the triplet $\mathcal{R}=\{\mathcal{E}, \mathcal{A}, \mathcal{P}\}$.

The exclusion sub-rule $\mathcal{E}$ dictates which channels are available. This sub-rule has the objective of preventing harmful interference to primary users. Exclusion sub-rules determine that channels currently occupied by primary users must be avoided by secondary users. They also determine how this 
interference avoidance is to be accomplished, e.g., through spectrum sensing, or geolocation databases. A survey on spectrum sensing is presented in [5]. Additional rules for exclusion also considered by regulators support the adoption of cooperative sensing for determining channel availability [2, 3]. A survey of cooperative sensing techniques and their tradeoffs has been captured in [6].

The channel assignment sub-rule $\mathcal{A}$ determines which channels can be used for opportunistic communications, a subset of the channels not excluded by $\mathcal{E}$. Regulation in [1] and [2] does not specify channel selection algorithms; however, it suggests that device manufacturers could use sensing information to select the best channel. If multiple secondary operators or devices seek to simultaneously use common spectra, efficient channel assignment will be important for maximizing channel reuse. A comparison of different channel assignment techniques for DSA has been examined in [7].

The power and transmission control sub-rule $\mathcal{P}$ dictates the maximum allowable power limits, transmit mask and techniques for minimizing power and interference. Power control is necessary in DSA applications to minimize interference among co-channel and adjacent channel users. These rules include maximum transmit power and Out of Band Emission (OOBE) requirements such as adjacent channel attenuation or Block Edge Mask (BEM) specifications. Power and transmission sub-rules can also work in tandem with exclusion sub-rules. For instance, the transmit power limits set by a BEM may take into account what systems currently operate in adjacent bands. Additionally, the maximum power limits of the secondary transmitters can also be reduced depending on the proximity to exclusion areas. Power control etiquette schemes have been proposed in [8,9].

\section{Decision Analysis}

In this section, we apply techniques from decision theory to explore regulators' objectives in setting spectrum access rules. In any decision process, there are objectives that the decision maker wishes to accomplish when selecting among alternatives. These objectives can be expressed as utility functions, which are used to evaluate the alternatives and make the final decision. In our formulation, distinct spectrum access rules are represented as alternatives available to the regulator, the decision maker, who must select the set of rules that maximizes a defined measure of social welfare.

\section{A. Objective Hierarchy}

The first and most fundamental objective of regulators is to enable telecommunications that are in the best interest of the public [1, 2]. In the context we consider here, this goal implies that regulators seek to create spectrum access rules as a means to support new and improved wireless services. Through spectrum access rules, regulators seek to maximize the spectrum efficiency of underutilized bands by accomplishing two sub-objectives: (1) minimizing harmful interference to primary operators' service; and (2) maximizing the utility of DSA spectrum for secondary operators. Managing the tradeoffs between these two sub-objectives is the main challenge regulators face in evaluating spectrum access rules. Ideally, spectrum access rules would allow for maximum secondary usage without creating any harmful interference to primary users.

We equate maximizing secondary operator utility with maximizing service revenue. This perspective is intuitive because customers demonstrate utility by paying for services. If secondary operators cannot provide suitable services with DSA spectrum, customers will not use these services. Maximizing revenue is captured in two sub-objectives: (1) maximizing service volume; and (2) minimizing customer churn. Maximizing service volume equates to supporting the highest volume of calls or data as possible. Customer churn is defined as the percentage of the customer base that leaves the service provider annually, usually as a result of service issues such as dropped or blocked calls [10-12]. When several secondary competitive operators share the DSA spectrum simultaneously, competition for resources could result in dropping existing service requests or blocking future attempts. Therefore, we express customer churn as a function of dropped or blocked services.

\section{B. Utility Model}

Generically, a multi-attribute utility model can be expressed as:

$$
u\left(x_{1}, x_{2}, \ldots, x_{n}\right)=\sum_{i=1}^{n} w_{i} u_{i}\left(x_{i}\right),
$$

where $x_{i}$ is the measure for attribute $i=1, \ldots, n, u_{i}(\cdot)$ is a single-attribute utility measure for attribute $i$ scaled in the interval $[0,1]$, and $w_{i}$ is the weight for measure $i$, with $\sum_{i} w_{i}=1$ [13]. Weights represent the perceived importance of a specific utility attribute.

Our multi-attribute utility model for evaluating spectrum access rules, derived from the objective hierarchy, is given by the following equation:

$$
\begin{aligned}
u_{\text {regulator }}= & \overbrace{w_{1} u_{p-\text { drop }}}^{\text {Primary Operator Utility }} \text { Secondary Operator Utility } \\
& +\overbrace{w_{2}[\underbrace{v_{1} u_{s-\text { bits }}}_{\text {Service Volume }}+\underbrace{v_{2}\left(z_{1} u_{s-\text { drop }}+z_{2} u_{s-\text { block }}\right)}_{\text {Customer Churn }}]}^{\text {. }} .
\end{aligned}
$$

The utility $u_{p-d r o p}$ represents the total utility for primary operators and is based on the service losses due to harmful secondary interference. The secondary operator utility is based on service volume and customer churn. Service volume is represented through the utility $u_{s-b i t s}$. Customer churn is represented through the two utility functions $u_{s-d r o p}$ and $u_{s-b l o c k}$, which consider the proportions of dropped and blocked service attempts, respectively. Weights $w_{i}, v_{i}$, and $z_{i}$ represent the relative importance between primary and secondary operators' utilities, service volume and customer churn, and drops and blocks, respectively. As in most policy considerations, relative weights are subjective and must be determined with appropriate input from stakeholders. 
The values of the $w_{i}$ reflect the relative importance the regulator attaches to services provided by primary and secondary operators. As primary user protection and prioritization is already reflected in the spectrum access rules (Section II), our study is agnostic on the type of service provided by primary and secondary operators and thus we set $w_{i}=w=0.5$. This value selection reflects that secondary service and primary service are equally valued. In other applications, the service value between primary and secondary service could be examined from a monetary or social benefit point of view to determine weights.

Determining the value of $v_{i}$ and $z_{i}$ should be driven by the perspective of network operators who provide secondary services. We obtain values of $v_{i}$ and $z_{i}$ through a technique known as Analytical Hierarchy Process (AHP) [4]. Using AHP, we interviewed two experts to perform pairwise comparisons between attributes of relevance to secondary operator performance in a DSA environment ${ }^{1}$. After determining which attribute is more important, the more important attribute receives a score from $1-9$, with 1 indicating that the two attributes are equally important. These pairwise comparisons are placed in matrix $\mathbf{A}$, with $a_{j i}=1 / a_{i j}$, where each row and column represents a specific attribute. Using the following equation:

$$
\mathbf{A w}=\lambda_{\max } \mathbf{w},
$$

and solving for $\lambda_{\max }$, the principal eigenvalue of $\mathbf{A}$, and $\mathbf{w}$, the principal right eigenvector of $\mathbf{A}$, we can normalize the entries of $\mathbf{w}$ by dividing by their sum and recover the weighted values for our utility function.

We repeated the above process twice, with inputs from our interviews with two experts in cellular network performance and obtained two perspectives for the weights $v_{i}$ and $z_{i}$. We asked each expert to compare the relative importance of minimizing customer churn versus maximizing service volume and minimizing session blocks versus minimizing session drops. The results of the interview are placed in a comparison matrix, from which the principal eigenvector is calculated. The results from this calculation and resulting weight values are shown in Table I. From Table I, we note that Expert A views existing service requests as more important than new service requests, whereas Expert B views new and existing service requests as equivalent.

\section{Evaluated Spectrum Access Rules}

In this study, we consider the intent of the FCC rules in $[1,16]$ and focus on channel exclusion techniques that consider energy detection thresholds and a geolocation database that defines exclusion areas. For power control, we propose an adaptive power control algorithm to minimize co-channel interference and conserve transmit power based on [17].

While more sophisticated spectrum sensing techniques exist, our evaluation considers spectrum sensing based on energy

\footnotetext{
${ }^{1}$ While in this paper we only examine only two viewpoints, we also note that group decision making and viewpoint aggregation has also been studied in $[14,15]$.
}

\begin{tabular}{|r|r|r|r|}
\hline Expert A & Blocks & Drops & $z_{i}$ \\
\hline Blocks & 1.00 & $1 / 6$ & 0.14 \\
\hline Drops & 6.00 & 1.00 & 0.86 \\
\hline \hline Expert A & Max Service & Min Churn & $v_{i}$ \\
\hline Max Service & 1.00 & $1 / 5$ & 0.17 \\
\hline Min Churn & 5.00 & 1.00 & 0.83 \\
\hline \hline Expert B & Max Service & Min Churn & \\
\hline Max Service & 1.00 & $1 / 7$ & 0.12 \\
\hline Min Churn & 7.00 & 1.00 & 0.88 \\
\hline
\end{tabular}

TABLE I

PAIRWISE COMPARISON MATRICES DERIVED FROM EXPERT INTERVIEWS AND UTILITY MODEL WEIGHTS FOR $z_{i}$ AND $v_{i}$. BLOCKS AND DROPS FOR EXPERT B ARE NOT CAPTURED SINCE $z_{i}=z=0.5$.

detection. Through energy detection, a channel $c$ is deemed available if the receiver of link $i$ measures the received power to be below the detection threshold $\alpha$, denoted as $I_{i}^{(c)}<\alpha$. Our study uses a detection threshold of $\alpha=-107 \mathrm{dBm}$ as indicated in [1]. Using sensing information, our work considers the channel assignment sub-rule through Least Interfering Channel (LIC) assignment, where the LIC is determined by the $\min _{c} I_{i}^{(c)}$. We also consider additional exclusion rules using cooperative sensing with hard combining. In hard combining, a channel is determined to be available if a certain proportion of the receivers detect power on the channel to be below the detection threshold.

Like the ruling in [1], we define channel exclusion through exclusion areas to protect primary operators from secondary interference. Secondary links in this exclusion model are not provided with protection areas and accept interference from one another. We consider exclusion areas surrounding primary links as disks of radius $d_{i, i}$, where $d_{i, j}$ is the Euclidean distance between the transmitter of link $i$ and receiver of link $j$. If primary link $i$ is using channel $c$, secondary link $j$ is permitted to use channel $c$ if $d_{j, i}>d_{i, i}$ and $d_{i, j}>d_{i, i}$. The distance $d_{i, i}$ can also be extended by additional factors. In our study, we define the exclusion areas such that $d_{j, i}>\kappa d_{i, i}$, with $\kappa \geq 1$ and we explore values $\kappa \in\{1,1.5,2,2.5\}$.

Power control is necessary in DSA applications to minimize interference among co-channel links. Additionally, changing network conditions require adjusting transmit power to maintain the requisite link Signal to Interference and Noise Ratio (SINR). Thus, we consider dynamic power control for primary and secondary users such that all links on channel $c, \mathcal{L}_{c}$, iteratively adjust their transmit power according to:

$$
p_{i}(k+1)=\min \left(p_{\max }, \frac{\beta}{\gamma_{i}} p_{i}(k)\right),
$$

where $k$ is the iteration number, $p_{i}$ is the power of transmitter $i$, and $\gamma_{i}$ is the SINR of link $i$. Foschini in [17] demonstrated that when transmitters use equation (4) to adjust their power levels, the transmit powers of the links will converge exponentially to an optimal power assignment. In our case, optimal power assignment means using only the amount of power necessary to maintain requisite link SINR. If the link cannot maintain an SINR of at least $\beta$ without the transmit power of the 


\begin{tabular}{|l|c|c|}
\hline & Sensing & Additional Channel Exclusion \\
\hline Rule 1 & Receiver Only & None \\
\hline Rule 2 & Receiver and Transmitter & None \\
\hline Rule 3 & Cooperative & Hard Combining Ratio $=.25$ \\
\hline Rule 4 & Cooperative & Hard Combining Ratio $=.5$ \\
\hline Rule 5 & Cooperative & Hard Combining Ratio $=1$ \\
\hline Rule 6 & Receiver Only & Exclusion space factor $=1$ \\
\hline Rule 7 & Receiver Only & Exclusion space factor $=1.5$ \\
\hline Rule 8 & Receiver Only & Exclusion space factor $=2$ \\
\hline Rule 9 & Receiver Only & Exclusion space factor $=2.5$ \\
\hline
\end{tabular}

TABLE II

Spectrum access RULeS for Simulations. Rules USE $\alpha=-107$ dBM and Least INTERFERING ChANNEL FOR CHANNEL ASSIGNMENT.

link exceeding maximum transmit power, $p_{\max }$, the link is infeasible and the power of the link is set to zero. Table II summarizes the list of the spectrum access rules that we consider in this study.

\section{System Model and Simulation Scenario}

In this section we introduce our system model, our simulation scenario, and our derivations of the utility functions. We adopt the SINR model for defining interference between co-channel links and determine which links are feasible. The simulation scenario defines how primary and secondary users share spectrum. This section closes with a description of how the utilities defined in Equation (2) are calculated from the simulation scenarios.

\section{A. System Model}

We define a set of frequency channels $C$ and a set of communication links $\mathcal{L}$. Each link $i \in \mathcal{L}$ comprises a transmitter and receiver, which seek to establish a wireless communications link using a channel $c \in C$. All $c \in C$ have a bandwidth of $W$. Given a set of communications links operating on a channel $c$, $\mathcal{L}_{c}$, the SINR of the receiver of link $i \in \mathcal{L}_{c}, \gamma_{i}^{(c)}$, is determined by:

$$
\gamma_{i}^{(c)}=\frac{g_{i i} p_{i}}{N_{o}+I_{i}^{(c)}},
$$

where $g_{j i}$ is the gain between the transmitter of link $j$ and the receiver of link $i$. The variable $p_{i}$ denotes the power of the transmitting node of link $i$, and $N_{o}$ the thermal noise. $I_{i}^{(c)}$ is the interference power at the receiver of link $i$, expressed as:

$$
I_{i}^{(c)}=\sum_{j \in \mathcal{L}_{c}, j \neq i} g_{j i} p_{j} .
$$

In this system model, a feasible link is a link whose receiver SINR, $\gamma_{i}$, is above a threshold $\beta$. We define a session as a pair of unidirectional links between communicating nodes and denote $\gamma_{i}$ as the SINR of the uplink and $\hat{\gamma}_{i}$ as the SINR of the downlink. A session is feasible if and only if the pair of links are both feasible, i.e., $\gamma_{i} \geq \beta$ and $\hat{\gamma}_{i} \geq \beta$.

\section{B. Simulation Scenario}

In our simulation, we consider a scenario in which a set of primary and secondary operators share a set of channels, $C$. Each operator comprises a set of access points and corresponding users associated with each access point. Access points are assumed to have established a control channel to coordinate channel assignment with the users. The simulation randomly places four primary and four secondary operator access points, each with twenty users surrounding the associated access points, in a square simulation area $(1000 \mathrm{~m} \times 1000 \mathrm{~m})$. After placement of the access points, users for each access point are randomly and uniformly placed within a distance $D_{\max }=$ $700 \mathrm{~m}$ from their respective access point. We assume a noise floor $\left(N_{o}\right)$ of $-110 \mathrm{dBm}, p_{\text {ref }}=p_{\max }=1 \mathrm{~W}$, path loss factor of 4 , and independent Rayleigh fading. To show a lower bound in our given scenario, our simulation considers a worst case scenario, where every link carries traffic and attempts to be in service simultaneously.

Initially, primary users are allowed to establish sessions with their corresponding access points using non-interfering channels $c \in C$. Following primary users, secondary sessions attempt to be admitted into the network individually and at random. Admission of secondary links begins by determining channel availability using exclusion sub-rule $\mathcal{E}$, followed by the channel assignment sub-rule $\mathcal{A}$, and then the power control and transmission sub-rule $\mathcal{P}$. In our scenario, we use each of the exclusion rules defined in Table II followed by LIC for channel assignment. We also assume that nodes are capable of perfect spectrum sensing and there exists a common control channel for exchange of sensing information. After channel assignment of link $i$ to channel $c$ (the LIC of the receiver) power control is initiated by the transmitter of secondary link $i$ with initial power parameter $p_{\text {ref }}$. Links in $\mathcal{L}_{c}$ then adjust their transmit power using equation (4), until the power settings of $\mathcal{L}_{c}$ converge. The simulation time $T$ is determined by using the simulation time step when link admission converges (no more links can be admitted) or when all links are attempted at least once, whichever comes last. Determining $T$ in this manner was done to allow every link to be attempted and simulations to converge.

\section{Utility Derivations}

The utility $u_{p-d r o p}$ is used to measure the primary service losses from harmful secondary interference. When secondary links are admitted, they may cause other sessions to become infeasible (harmful interference) through lowering the SINR of the co-channel links below $\beta$. If the transmitters of those links cannot maintain an SINR of $\beta$ without exceeding $p_{\text {max }}$, those links will drop. Thus, $u_{p-d r o p}$ is a linear utility function that is zero if all sessions are dropped during $T$ and reaches a value of 1 if no primary sessions are dropped.

Similarly, the utility function $u_{s-d r o p}$ is based on the number of dropped sessions for the secondary operators in the same manner. However, in this case the linearly decreasing function reaches zero when the percentage of secondary session drops reaches $4.5 \%$ [18]. We use $4.5 \%$ as an expected worst case. Thus, $u_{s-d r o p}$ is a linear utility function that is one if no secondary sessions are dropped and zero if more than $4.5 \%$ links are dropped.

To develop $u_{s-b i t s}$, the utility for the attribute bit volume, we examine secondary link feasibility over a time period $T$. This is a linear utility function that is zero if no secondary 


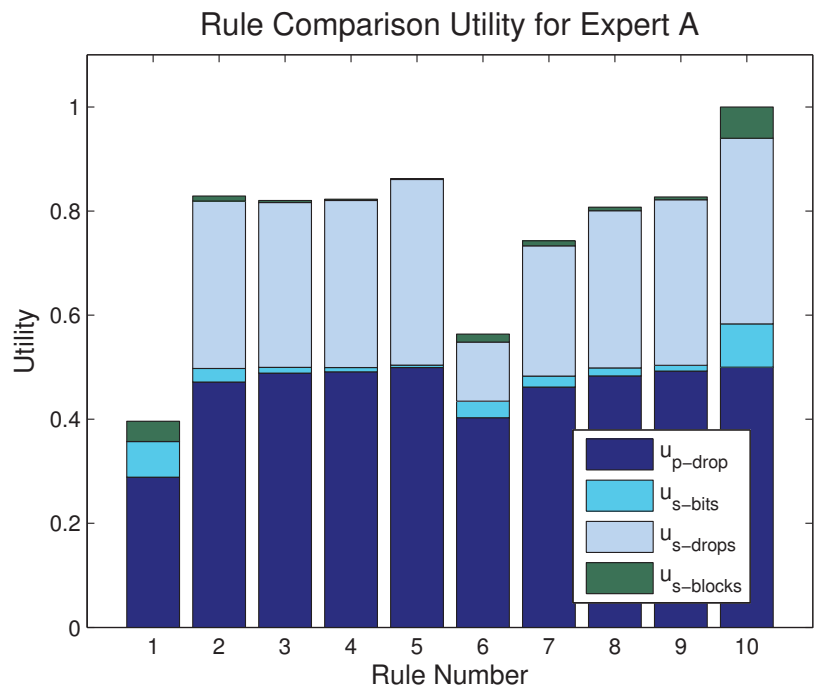

Fig. 1. Utility comparison of spectrum access rules using attribute weights from Expert A. Rule 10 shows the utopia point for perspective.

sessions are feasible and reaches a value of 1 if all possible secondary session are feasible during time period $T$.

The utility $u_{s-b l o c k}$ represents the measure of blocked secondary session admissions. During admission, secondary users can be blocked for two reasons. First, the channel could be unavailable because of exclusion, i.e., the spectrum access subrule $\mathcal{E}$ prevents the channel from being used. Second, the requisite SINR of the link may not be reached because of excessive co-channel interference. This linear utility function is zero if all secondary links are blocked and reaches a value of 1 if no links are blocked.

\section{Results}

Figures 1 and 2 show the resulting utility for attribute weights from Expert A and Expert B, respectively. Rule 10 represents the utopia point, the maximum utility due to each attribute if there were no conflicts between primary and secondary user objectives. The most salient feature in both Figures 1 and 2 is the poorest performing rule, Rule 1. Placing this in context, Rule 1's exclusion sub-rules are based only on receiver sensing, i.e. channels are available if the measured power is below a detection threshold. The poor performance is due to two reasons. First, Rule 1 provides insufficient protection for primary users, causing a relatively large proportion of primary sessions to drop, i.e. low utility for $u_{p-d r o p}$. Second, Rule 1 also causes many secondary links to drop, i.e. low utility for $u_{s-d r o p}$. Thus, Rule 1 , the receiveronly sensing exclusion sub-rule, provides the least amount of utility for primary and secondary operators, at the expense of admitting a large number of new sessions.

Figures 1 and 2 show that Rules 2, 5, and 9 result in the highest overall utilities. Rule 2 and Rule 5 both use cooperative sensing by hard combining. Rule 2 requires the transmitter of the link to sense the LIC of the receiver to be below the detection threshold. Compared to receiver-only sensing, this is a dramatic improvement in providing protection from harmful

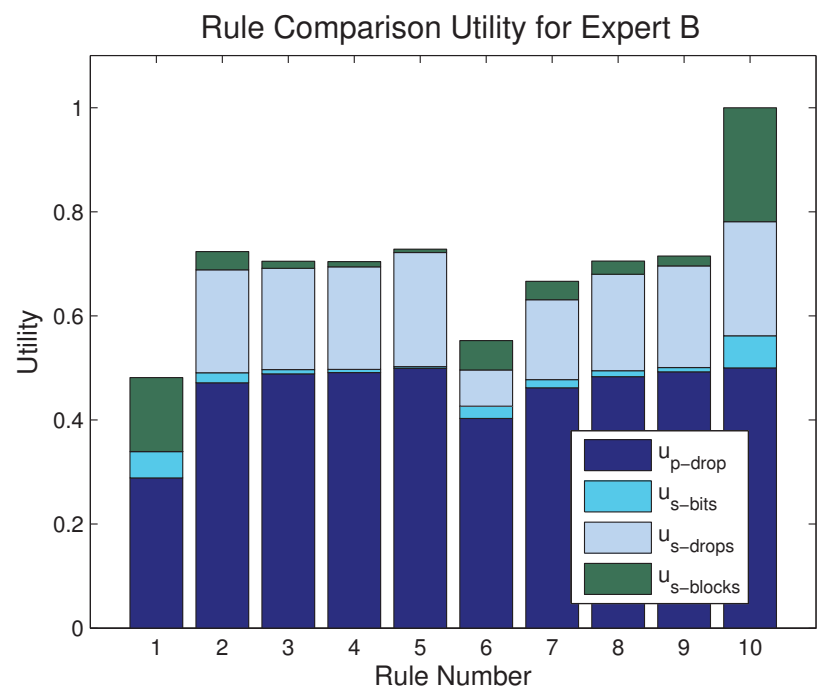

Fig. 2. Utility comparison of spectrum access rules using attribute weights from Expert B. Rule 10 shows the utopia point for perspective.

interference to both primary and secondary users, i.e. high $u_{s-d r o p}$ and $u_{p-d r o p}$. Rule 5 only allows channels to be available if all users associated to the same access point sense the LIC of the receiver to be below the detection threshold, providing the best protection for primary and secondary users, i.e. $u_{s-d r o p}$ and $u_{p-d r o p}$. However, Rule 5 provides very little throughput for secondary users, i.e. low $u_{s-b i t s}$ and low $u_{s-b l o c k}$, by being overly conservative about opportunistic use. Rule 9 provides the largest exclusion regions, allowing for significant amount of protection for primary users through preventing secondary use. In summary, based on the two weighted values from Experts $\mathrm{A}$ and B, Rules 2, 5, and 9 have nearly equivalent overall utilities. It is also evident that as secondary users are less restricted and achieve higher $u_{s-b i t s}$ and $u_{s-b l o c k}$, they can cause more service drops to both primary and secondary users and thus lower overall utilities. This phenomenon is magnified in the utility function since throughput, $u_{s-b i t s}$, is less important in the perspective of both experts.

We next examine the causes of blocking. Figure 3 shows the cumulative number of blocks for each rule during the simulation and classifies them according to the cause of session blocking. An SINR Block is a session block caused because the SINR of the link cannot be reached. SINR Blocks are a result of limited transmitter power or high levels of interference. Channel Access Blocks are due to the exclusion sub-rule, preventing access to the spectrum. From Figure 3, the rules with the highest utilities also have the highest proportions of channel access blocks. One could also argue that Channel Access Blocks are less expensive than SINR Blocks, in that on the latter the transmitter must use energy and time to attempt to access the channel.

\section{Conclusion and Future Work}

Applying decision theory, this study created a multi-attribute utility model for evaluating different dynamic spectrum access rules. Using this utility model, we developed a scenario for 


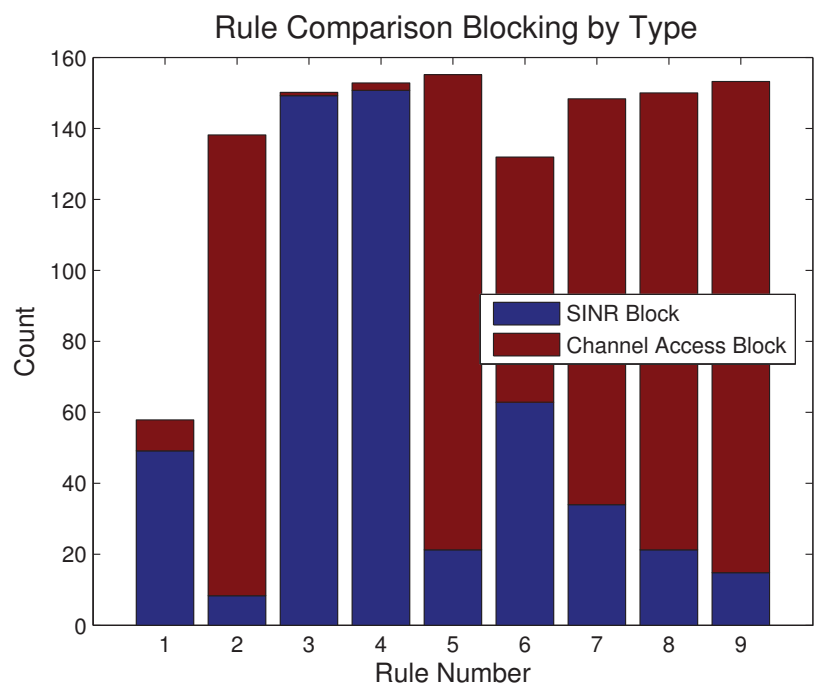

Fig. 3. Blocking by type of rules. Rules 2, 5, and 9 have the highest proportions of channel access blocks.

evaluating network performance of both secondary and primary operators and evaluated different spectrum access rules. We considered rules based on sensing and exclusion spaces. Our results show that as secondary users are less restricted, they can cause service drops to both primary and secondary users and thus overall lower utilities. Additionally, rules with higher proportion of channel access blocks result in the largest overall utilities.

Future work will examine the risk preferences of regulators and secondary network operators. This paper considered riskneutral operators and regulators, which led us to use linear utility functions. A new multi-attribute utility with risk-prone or risk-averse preferences would result in convex or concave utility functions, respectively [19]. Additionally, it is not clear that the attributes throughput and customer churn should be modeled as being independent. For instance, if there is no throughput there are no drops due to the non-existence of links performing service. Thus, investigation into a utility model which is not utility-independent should also be examined. In conclusion, this work provides a first look in developing a decision analysis framework for regulators to evaluate the technical merits of spectrum access rules.

\section{REFERENCES}

[1] Federal Communications Commission, " In the Matter of: Unlicensed Operation in the TV Broadcast Bands (ET Docket No. 04-186) and Additional Spectrum for Unlicensed Devices Below $900 \mathrm{MHz}$ and in the $3 \mathrm{GHz}$ Band (ET Docket No. 02-380)," FCC 10-174:Second Memorandum Opinion and Order, September 2010.

[2] Ofcom, "Implementing geolocation," Consultation, November 2010.

[3] Federal Communications Commission, "10-198:In the Matter of: Promoting More Efficient Use of Spectrum Through Dynamic Spectrum Use Technologies (ET
Docket No. 10-237)," FCC 10-198: Notice of Inquiry, November 2010.

[4] T. Saaty, "How to make a decision: the analytic hierarchy process," European Journal of Operational Research, vol. 48, pp. 9-26, 1990.

[5] T. Yucek and H. Arslan, "A survey of spectrum sensing algorithms for cognitive radio applications," IEEE Communications Surveys Tutorials, 2009.

[6] S. Mishra, A. Sahai, and R. Brodersen, "Cooperative sensing among cognitive radios," IEEE International Conference Communications (ICC), vol. 4, 2006.

[7] J. Deaton, S. Ahmad, U. Shukla, R. Irwin, L. DaSilva, and A. MacKenzie, "Evaluation of dynamic channel and power assignment for cognitive networks," Springer Journal on Wireless Personal Communications, vol. 57, 2011.

[8] D. Satapathy and J. Peha, "A novel co-existence algorithm for unlicensed fixed power devices," IEEE Wireless Communications and Networking Conference (WCNC), vol. 3, 2000.

[9] _ - "A novel co-existence algorithm for unlicensed variable power devices," IEEE International Conference on Communications (ICC), 2001.

[10] J.D. Power and Associates, "Incidence of Dropped Calls Increases Considerably among Customers Who Are Most Likely to Switch Wireless Providers," September 2010.

[11] M. Lombardo, personal communication.

[12] M. Shomaker, personal communication.

[13] W. Edwards, R. Miles, and D. Von Winterfeldt, Advances in Decision Analysis. Citeseer, 2007.

[14] E. Forman and K. Peniwati, "Aggregating individual judgments and priorities with the analytic hierarchy process," Elsevier European Journal of operational research, vol. 108, no. 1, 1998.

[15] R. Ramanathan and L. Ganesh, "Group preference aggregation methods employed in AHP: An evaluation and an intrinsic process for deriving members' weightages,' Elsevier European Journal of Operational Research, vol. 79, no. 2, 1994.

[16] Federal Communications Commission, “ In the Matter of: Unlicensed Operation in the TV Broadcast Bands (ET Docket No. 04-186) and Additional Spectrum for Unlicensed Devices Below $900 \mathrm{MHz}$ and in the $3 \mathrm{GHz}$ Band (ET Docket No. 02-380)," FCC 08-260: Second Report and Order and Memorandum Opinion and Order, November 2008.

[17] G. Foschini and Z. Miljanic, "Distributed autonomous wireless channel assignment algorithm with power control," IEEE Transactions on Vehicular Technology, vol. 44, no. 3, pp. 420-429, August 1995.

[18] A. Golub and P. Carton, "The Battle Continues Among Wireless Industry Leaders," ChangeWave Area Report, May 2010.

[19] R. Keeney and H. Raiffa, Decisions with multiple objectives. Cambridge University Press, 1993. 